Rev. Int. Contam. Ambie. 34 (1) 45-55, 2018

DOI: 10.20937/RICA.2018.34.01.04

\title{
TOXICIDAD AGUDA DIFERENCIAL DE TALSTAR® (BIFENTRINA) Y BIOTHRINE® (DELTAMETRINA) EN LA TILAPIA NILÓTICA Oreochromis niloticus
}

\author{
Juan José SANDOVAL GÍO ${ }^{1}$, Luis Enrique CASTILLO SÁNCHEZ * \\ Eduardo Alfredo ZARZA MEZA², José Manuel HERNÁNDEZ JIMÉNEZ ${ }^{1}$, \\ Javier Hernán FERNÁNDEZ SERRANO ${ }^{1}$ y Agustín PINEDA DOPORTO ${ }^{1}$
}

${ }^{1}$ Instituto Tecnológico de Tizimín, Tecnológico Nacional de México. Final aeropuerto Cupul, Tizimín, Yucatán, México, C. P. 97700

${ }^{2}$ Facultad de Ciencias Biológicas y Agropecuarias, Universidad Veracruzana. Región Poza-Rica-Tuxpan. Carretera Tuxpan-Tampico, km 7.5, Colonia Universitaria. Tuxpan, Veracruz, México, C. P. 92850

*Autor para correspondencia: hymenopteralecs@hotmail.com

(Recibido abril 2016; aceptado mayo 2017)

Palabras clave: acuacultura, insecticidas, peces, CL50, dengue, ecotoxicología

\section{RESUMEN}

La tilapia Oreochromis niloticus es una especie de importancia en acuacultura con un desarrollo promisorio en la península de Yucatán. La bifentrina y la deltametrina son dos piretroides ampliamente utilizados por el sector salud en Yucatán para el control del mosquito Aedes aegypti, vector del dengue. Se evaluó la toxicidad aguda diferencial de Talstar ${ }^{\circledR}$ (sustancia activa, bifentrina) y Biothrine ${ }^{\circledR}$ (principio activo, deltametrina), en juveniles de tilapia $O$. niloticus. Se usaron ocho concentraciones de Talstar ${ }^{\circledR}$ $(2.5-400 \mu \mathrm{g} / \mathrm{L})$ y ocho concentraciones de Biothrine ${ }^{\circledR}(0.56-112 \mu \mathrm{g} / \mathrm{L})$, más un testigo sin insecticida y tres repeticiones por tratamiento. Los signos de toxicidad en los peces bajo la influencia de ambos insecticidas fueron: nadadas erráticas, desbalance e hiperventilación, aunque resultaron más evidentes y en un menor tiempo de aparición, en las concentraciones más altas. El análisis probit reveló que la concentración letal media (CL50) de Talstar ${ }^{\circledR}$ fue de $75.11 \mu \mathrm{g} / \mathrm{L}$ a las 96 horas con intervalos de confianza (95\%) de 58.76 (inferior) y 96.34 (superior) $\mu \mathrm{g} / \mathrm{L}$. La CL50 de Biothrine ${ }^{\circledR}$ fue de $28.36 \mu \mathrm{g} / \mathrm{L}$ a las 96 horas, con intervalos de 23.29 y $35.27 \mu \mathrm{g} / \mathrm{L}$. Estos valores son considerados como extremadamente tóxicos y alertan por el efecto contaminante potencial que podrían producir en ambientes naturales y de acuacultura. Es necesario continuar con la evaluación de los mecanismos de toxicidad de insecticidas que son utilizados en el sector salud para especies de importancia acuícola en México. Lo anterior por la perspectiva de continuar con su empleo y por el desarrollo del sector en la península de Yucatán.

Key words: aquaculture, insecticides, fishes, LC50, dengue, ecotoxicology

\begin{abstract}
Tilapia, Oreochromis niloticus is an aquacultural important species and both, bifenthrin and deltamethrin are two synthetic pyrethroids used by Health Services in Yucatán to control Aedes aegypti mosquito, dengue's vector. Differential acute toxicity of Talstar ${ }^{\circledR}$
\end{abstract}


(bifenthrin) and Biothrine ${ }^{\circledR}$ (deltamethrin), in tilapia O. niloticus was evaluated. Eight concentrations of Talstar ${ }^{\circledR}(2.5-400 \mu \mathrm{g} / \mathrm{L})$, and eight concentrations of Biothrine ${ }^{\circledR}$ $(0.56-112 \mu \mathrm{g} / \mathrm{L})$, were used, along with a control treatment without insecticide, and three repetitions for each one. Signs of toxicity in fishes under influence of these insecticides were: erratic swimming, loss of balance and hyperventilation, especially in highest concentrations. Probit analysis showed a lethal medium concentration (LC50) at 96 hours for Talstar ${ }^{\circledR}$ of $75.11 \mu \mathrm{g} / \mathrm{L}$. Confidence limits (95\%) were 58.76 and 96.34 $\mu \mathrm{g} / \mathrm{L}$. LC50 at 96 hours for Biothrine ${ }^{\circledR}$ was $28.36 \mu \mathrm{g} / \mathrm{L}$, confidence limits $(95 \%)$, were 23.29 and $35.27 \mu \mathrm{g} / \mathrm{L}$ ). These values are considered as extremely toxic, and they should be observed with alert because they could have potential toxic effect in both, natural or aquaculture environments. It is needed to continue the evaluation of the acute and chronic toxicity mechanisms of insecticides used in the health sector for aquaculture species in México. This, because the perspectives of use of these substances and the development of aquaculture in the Yucatan peninsula.

\section{INTRODUCCIÓN}

La acuacultura en México se ha desarrollado notablemente, en 2013 aportó $38 \%$ del valor comercial de la producción pesquera nacional, equivalente a unos 475 millones de dólares. La tilapia Oreochromis niloticus ha alcanzado un primer lugar entre las especies piscícolas mexicanas con 15 mil ha disponibles para engorda en todo el territorio nacional (CONAPESCA 2013).

La península de Yucatán representa una zona de posicionamiento para la acuacultura mexicana, especialmente por su clima apropiado y su ubicación estratégica para el mercado internacional (Olvera 2010). Sin embargo, en esta región predomina un suelo cárstico muy permeable (Pérez y Pacheco 2004), lo que pone en peligro la sustentabilidad acuícola por la vulnerabilidad del acuífero a los contaminantes. Esto debido a que gran parte de la región posee fuentes subterráneas de agua y las fracturas verticales del subsuelo hace que los contaminantes químicos y microbiológicos presentes en el ambiente, fluyan por lixiviación de una manera más rápida y directa hacia el nivel freático (Buckley et al. 1994, Pacheco-Avila et al. 2004). Esta exposición del recurso hídrico a contaminantes provenientes de diversas fuentes, entre ellas del sector salud, pone en riesgo la inocuidad alimentaria (Sabra y Mehana 2015).

El dengue ocupa desde 2005 el cuarto lugar entre las enfermedades infecciosas prevalentes en México, con regiones de alta susceptibilidad como la península de Yucatán (Altagracia et al. 2012, Barba 2014). En Yucatán, para 2011 y 2012 se superaron los 5000 casos, por lo que esta zona se considera actualmente hiperendémica para esta enfermedad (Salomón et al. 2012, González 2013).
El control del vector del dengue, el mosquito Aedes aegypti, se lleva a cabo por fumigación con diversos piretroides, especialmente con formulaciones comerciales. Como resultado de estas fumigaciones se pueden contaminar cuerpos de agua y por consiguiente, la biodiversidad que ahí habita (Akan et al. 2015).

Se ha documentado que diversos grupos de organismos (no blanco), tanto terrestres como acuáticos, pueden resentir el impacto a la exposición de plaguicidas. Ejemplos de éstos son los insectos acuáticos (Antwi y Reddy 2015), abejorros (Gradish et al. 2012) y mamíferos marinos (Alonso et al. 2012). A pesar de que actualmente hay mucha información sobre el impacto de la exposición a plaguicidas en diferentes grupos de organismos, el impacto negativo en organismos no blanco pudiera estar subestimado (Velíšek et al. 2009a, González 2013).

Actualmente, los piretroides bifentrina y deltametrina, se encuentran en la lista de productos recomendados por el sector salud en México para el combate de insectos vectores de enfermedades (CENAPRECE 2016). En Yucatán, desde 2007, la secretaría de salud ha fumigado continuamente para hacer frente a brotes de dengue, con las fórmulas comerciales Talstar ${ }^{\circledR}$ y Biothrine ${ }^{\circledR}$, a base de bifentrina y deltametrina, respectivamente, dos de los insecticidas empleados por ese sector (SSA 2010, Jahan y Sadiq 2012, González 2013).

La bifentrina [2-metilbifenil-3-ilmetil (Z)-(1RS, 3RS)-3-(2-cloro-3,3,3-trifluoroprop-1-enil)-2,2-dimetilciclopropanocarboxilato] es un piretroide tipo I, que en los últimos años se ha incrementado su uso, tanto doméstico, como en el control de plagas en agricultura o de vectores en el sector salud (Brander et al. 2016).

La deltametrina [(S)-a-ciano-3-fenoxibenzil (1 R,3R)-3-(2,2-dibromvinil)-2,2-dimetilciclo propano-carboxilato], se clasifica como piretroide 
tipo II y actualmente presenta un amplio uso doméstico, agrícola y para el control de vectores (Prusty et al. 2015, Brander et al. 2016). Se tiene registrado que la deltametrina causó mortandades masivas de Anguilla anguilla en el lago Ballaton, Hungría en los veranos de 1991 y 1995, luego de haberse aplicado este plaguicida en repetidas ocasiones para el control de mosquitos (Kidd y James 1991, Bálint et al. 1995).

En este contexto, las investigaciones sobre el efecto de insecticidas en peces de importancia para la acuacultura aún es escasa (Weston et al. 2011). Esto es relevante al haberse demostrado que los piretroides sintéticos pueden ser tóxicos para los peces (Dobsikova et al. 2006, Sarikaya 2009).

Aunque existen numerosas investigaciones que han documentado los efectos tóxicos agudos o subagudos de la bifentrina y de la deltametrina en peces de importancia comercial, éstos se orientan al estudio en la sustancia en grado técnico y no en formulaciones comerciales (Boateng et al. 2006, Velíšek et al. 2007, Karasu et al. 2009, Hedayati et al. 2012). Yildirim et al. (2006), consideran que las evaluaciones de toxicidad utilizando sólo los principios activos son insuficientes, por lo que deben incluir determinaciones de los efectos tóxicos de las formulaciones comerciales para estimar la acción sinérgica de los aditivos que contienen.

Dadas las perspectivas promisorias de desarrollo de la actividad acuícola en diversas zonas de la península de Yucatán, se requiere realizar investigaciones para conocer el potencial riesgo tóxico de insecticidas en peces de importancia comercial, tanto de los principios activos como de las formulaciones comerciales utilizadas por el sector de salud pública (Dobsikova et al. 2006, de Moraes et al. 2013, Chandrasekara y Premarathna 2016).

Este es el primer trabajo donde se evalúan los efectos tóxicos agudos individuales de Talstar ${ }^{\circledR}$ (a base de bifentrina) y Biothrine ${ }^{\circledR}$ (principio activo, deltametrina) sobre estadios juveniles de la tilapia $O$. niloticus, usando como criterio de determinación la concentración letal media (CL50).

\section{MATERIALES Y MÉTODOS}

Ochocientas tilapias juveniles de la especie $O$. niloticus (longitud total: $6.34 \pm 0.81 \mathrm{~cm}$, peso: 5.14 $\pm 0.65 \mathrm{~g})$, masculinizadas con $17 \alpha$-metil testosterona, se adquirieron en CINVESTAV-Mérida y se aclimataron por 30 días. Los organismos fueron distribuidos en cuatro tinas de plástico de $1000 \mathrm{~L}$, en el área acuícola del Instituto Tecnológico de Tizimín,
Yucatán, México. El suministro de aire fue constante y se monitoreó la temperatura $\left(\mathrm{T}^{\circ}\right)$, el oxígeno disuelto $\left(\mathrm{O}_{2}\right)$ y el $\mathrm{pH}$ del agua diariamente con un medidor multiparámetro portátil (Hach, modelo Senson 156). Cada quince días, se tomaron muestras de agua por triplicado, para determinar la dureza total, el amonio y los nitritos. A los peces se les suministró alimento comercial (Tilapia Chow ${ }^{\circledR}, 30 \%$ de proteína) ad libitum.

Finalizada la aclimatación, se seleccionaron al azar 540 tilapias para realizar dos bioensayos estáticos de 96 h (Reish y Oshida 1987). Cada uno de los tratamientos constó de 10 peces (longitud total $=8.07 \pm 1 \mathrm{~cm}$, peso $=11.2 \pm 1.61 \mathrm{~g}$ ) por pecera de $120 \mathrm{~L}$, por triplicado.

Los peces no se alimentaron desde dos días antes y durante todo el bioensayo para evitar presencia de heces. Se registró la temperatura, el oxígeno disuelto y el $\mathrm{pH}$ de las peceras cada hora durante las primeras doce horas y después cada seis horas hasta el término de cada bioensayo.

\section{Preparación de las soluciones y bioensayo pre- liminar}

Las sustancias a evaluar se compraron en un negocio local de productos agropecuarios. Se preparó una solución reserva con las indicaciones del productor, tal cual se presentaron en las hojas de seguridad. Por separado se disolvieron en agua destilada los polvos a hidratar de Talstar® (bifentrina) y Biothrine ${ }^{\circledR}$ (deltametrina), respectivamente, mismos que se colocaron en contenedores de plástico de $10 \mathrm{~L}$. Se tomó una muestra de $10 \mathrm{~mL}$ para determinar su concentración por espectrometría y las soluciones a evaluar se prepararon por diluciones repetidas.

Antes de realizar el bioensayo de evaluación, se llevó a cabo una prueba de intervalo de toxicidad, esto es un bioensayo preliminar, en el que se tomaron como referencia los valores registrados en la literatura para estas sustancias.

Cabe destacar que al estar los insecticidas utilizados en forma de polvos para hidratar, así como al haber preparado las disoluciones en agua destilada, no fue necesario incluir en los tratamientos un testigo solvente, solamente un testigo agua (negativos, sin insecticida).

\section{Bioensayo 1: Talstar ${ }^{\circledR}$}

La sustancia analizada fue Talstar ${ }^{\circledR}$ con bifentrina (9\%) como principio activo y $91 \%$ de ingredientes surfactantes y solventes. Se evaluaron ocho concentraciones $(2.5-400 \mu \mathrm{g} / \mathrm{L})$ con tres repeticiones cada una. Se utilizó un grupo testigo de agua destilada, sin insecticida (Cuadro I). 


\section{Bioensayo 2: Biothrine ${ }^{\circledR}$}

Se utilizó Biothrine ${ }^{\circledR}$ con $2.5 \%$ de deltametrina como principio activo y $97.5 \%$ de ingredientes surfactantes y solventes para evaluar ocho concentraciones $(0.56-112 \mu \mathrm{g} / \mathrm{L})$, con tres repeticiones cada una, así como un grupo testigo negativo, al que se le agregó agua destilada (Cuadro I).

Durante los bioensayos se observaron y documentaron los cambios en el comportamiento de los organismos, se registró la hora y el porcentaje de mortalidad, la que se definió como la ausencia de movimiento en branquias y aletas en respuesta a estímulos externos (Zapata y Pedrero 2008). La CL50 se obtuvo mediante el método probit (Finney 1971), con el programa Statgraphics Plus version 5.1.

\section{RESULTADOS}

No se observó mortalidad en los peces de los grupos testigo negativo en ambos bioensayos. La temperatura y el $\mathrm{pH}$ de los acuarios no variaron significativamente, sólo el oxígeno disuelto presentó una disminución no significativa $(\mathrm{p} \leq 0.05)$ en la mayor concentración agregada del insecticida. Los valores de la calidad del agua (media \pm D.E.) se muestran en el cuadro II.

\section{Bioensayo 1: Talstar ${ }^{\circledR}$}

En la evaluación de Talstar $\AA_{\text {, }}$ se observó que los peces nadaban de manera errática y disfuncional a los pocos minutos de haber administrado la mezcla comercial del piretroide bifentrina adicionado con solventes y emulsificantes, especialmente en las mayores concentraciones. La mortalidad se presentó en las dosis más altas en tiempos más cortos. Por ejemplo, para la concentración de $5 \mu \mathrm{g} / \mathrm{L}$ la mortalidad ocurrió a partir de las 84 horas de exposición. En contraste con la concentración más alta $(400 \mu \mathrm{g} / \mathrm{L})$, en la que la mortalidad inició en la primer hora de exposición al piretroide (Cuadro III).

CUADRO III. TABLA DE MORTALIDAD DE Oreochromis niloticus POR EFECTO DE TALSTAR ${ }^{\circ}$ A DIFERENTES HORAS DE LECTURA

\begin{tabular}{lrrrrrrrr}
\hline $\begin{array}{c}\text { Tiempo } \\
(\mathrm{h})\end{array}$ & \multicolumn{7}{c}{ Concentración de Talstar® $(\mu \mathrm{g} / \mathrm{L})$} \\
\cline { 2 - 8 } & 2.5 & 5.0 & 10 & 25 & 50 & 100 & 200 & 400 \\
\hline 0.5 & 0 & 0 & 0 & 0 & 0 & 0 & 0 & 0 \\
1 & 0 & 0 & 0 & 0 & 0 & 0 & 13 & 25 \\
2 & 0 & 0 & 0 & 0 & 0 & 0 & 25 & 50 \\
3 & 0 & 0 & 0 & 0 & 0 & 0 & 38 & 75 \\
4 & 0 & 0 & 0 & 0 & 0 & 0 & 50 & 100 \\
5 & 0 & 0 & 0 & 0 & 0 & 13 & 63 & 100 \\
6 & 0 & 0 & 0 & 0 & 0 & 25 & 75 & 100 \\
7 & 0 & 0 & 0 & 0 & 13 & 38 & 88 & 100 \\
8 & 0 & 0 & 0 & 0 & 13 & 50 & 100 & 100 \\
9 & 0 & 0 & 0 & 0 & 25 & 63 & 100 & 100 \\
10 & 0 & 0 & 0 & 0 & 25 & 75 & 100 & 100 \\
11 & 0 & 0 & 0 & 0 & 25 & 88 & 100 & 100 \\
12 & 0 & 0 & 0 & 0 & 38 & 100 & 100 & 100 \\
18 & 0 & 0 & 0 & 0 & 38 & 100 & 100 & 100 \\
24 & 0 & 0 & 0 & 13 & 50 & 100 & 100 & 100 \\
36 & 0 & 0 & 0 & 13 & 50 & 100 & 100 & 100 \\
48 & 0 & 0 & 13 & 13 & 50 & 100 & 100 & 100 \\
60 & 0 & 0 & 13 & 25 & 50 & 100 & 100 & 100 \\
72 & 0 & 0 & 13 & 25 & 50 & 100 & 100 & 100 \\
84 & 0 & 13 & 25 & 25 & 63 & 100 & 100 & 100 \\
96 & 0 & 13 & 25 & 25 & 63 & 100 & 100 & 100 \\
\hline
\end{tabular}

CUADRO I. CONCENTRACIONES NOMINALES $(\mu \mathrm{g} / \mathrm{L})$ DE TALSTAR ${ }^{\circ}$ Y BIOTHRINE® EVALUADAS EN Oreochromis niloticus

\begin{tabular}{|c|c|c|c|c|c|c|c|c|c|}
\hline Insecticida & $\mathrm{C}$ & $\mathrm{T} 1$ & $\mathrm{~T} 2$ & $\mathrm{~T} 3$ & $\mathrm{~T} 4$ & $\mathrm{~T} 5$ & T6 & T7 & T8 \\
\hline Talstar ${ }^{\circledR}$ & 0 & 2.5 & 5.0 & 10.0 & 25.0 & 50.0 & 100.0 & 200.0 & 400.0 \\
\hline Biothrine ${ }^{\circledR}$ & 0 & 0.56 & 1.4 & 2.8 & 7.0 & 14.0 & 28.0 & 56.0 & 112.0 \\
\hline
\end{tabular}

$\mathrm{C}=$ grupo testigo negativo, $\mathrm{T}=$ tratamiento

CUADRO II. PARÁMETROS FÍSICOS Y QUÍMICOS DEL AGUA EVALUADOS EN LOS BIOENSAYOS DE Oreochromis niloticus EXPUESTOS A TALSTAR ${ }^{\circledR}$ Y BIOTHRINE®

\begin{tabular}{cccccc}
\hline $\mathrm{T}^{\mathrm{a}}\left({ }^{\circ} \mathrm{C}\right)$ & $\begin{array}{c}\mathrm{O}_{2} \\
(\mathrm{mg} / \mathrm{L})\end{array}$ & $\mathrm{pH}:$ & amonio $(\mathrm{mg} / \mathrm{L})$ & nitratos: ppm & $\begin{array}{c}\text { dureza total: } \\
(\mathrm{CaCO} 3 \mathrm{mg} / \mathrm{L})\end{array}$ \\
\hline $25 \pm 2$ & $5.87 \pm 0.76$ & $6.9 \pm 0.9$ & $0.079 \pm 0.019$ & $2.07 \pm 1.02$ & $360 \pm 68$ \\
\hline
\end{tabular}


El análisis probit $\left(\mathrm{p} \leq 0.01, \mathrm{X}^{2}=110.86\right)$ reveló que la CL50 de Talstar ${ }^{\circledR}(9 \%$ de bifentrina, como ingrediente activo) en $O$. niloticus fue de $75.11 \mu \mathrm{g} / \mathrm{L}$ a las 96 horas. Los intervalos de confianza menor y mayor al $95 \%$ fueron 58.76 y $96.34 \mu \mathrm{g} / \mathrm{L}$, respectivamente (Fig. 1, Cuadro IV).

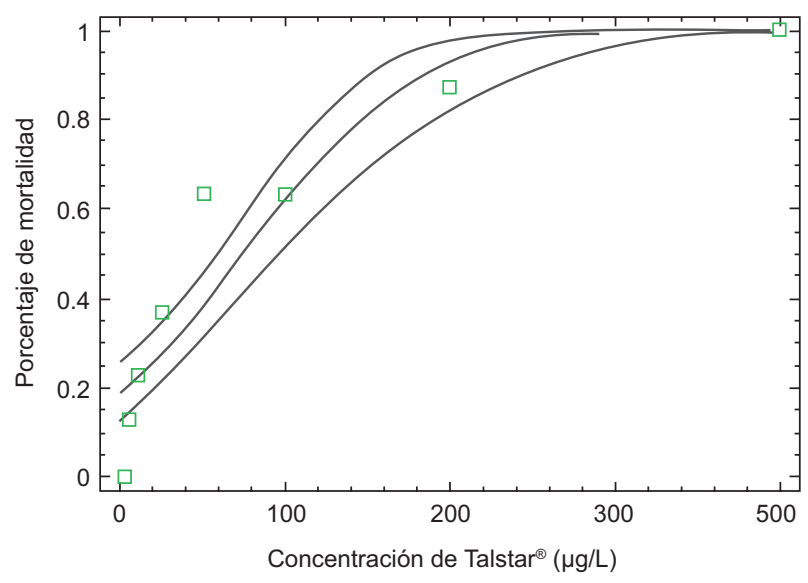

Fig. 1. Curva de la concentración letal 5096 h (I.C. $95 \%=$ 58.77-96.34, $\left.\mathrm{p}<0.01, \mathrm{R}^{2}=82.07, x^{2}=110.86\right)$ para Talstar ${ }^{\circledR}$ en Oreochromis niloticus. I.C. $=$ Intervalo de confianza, $\mathrm{R}^{2}=$ coeficiente de determinación, $x^{2}=$ chi cuadrada

\section{Bioensayo 2: Biothrine ${ }^{\circledR}$}

En la evaluación de Biothrine ${ }^{\circledR}$, también se observó que los peces nadaban de manera errática y disfuncional a los pocos minutos de haber administrado la mezcla comercial, especialmente en las mayores concentraciones. De igual modo que en Talstar ${ }^{\circledR}$, la mortalidad se presentó en las dosis más altas en tiempos más cortos, mientras que para las dosis menores, la mortalidad sucedió en un tiempo mayor. Por ejemplo, para las dos concentraciones más bajas $(0.56$ y $1.4 \mu \mathrm{g} / \mathrm{L})$ no se presentó mortalidad. Respecto a la concentración de $2.8 \mu \mathrm{g} / \mathrm{L}$, la mortalidad ocurrió a partir de las 84 horas de exposición. En contraste, en la concentración más alta $(112 \mu \mathrm{g} / \mathrm{L})$, la mortalidad inició en la segunda hora de exposición al piretroide (Cuadro V).

El análisis probit $\left(\mathrm{p} \leq 0.01, \mathrm{X}^{2}=127.1\right)$ reveló que la CL50 de Biothrine ${ }^{\circledR}(2.5 \%$ de deltametrina, como ingrediente activo) en $O$. niloticus fue de $28.36 \mu \mathrm{g} / \mathrm{L}$ a las 96 horas. Los intervalos de confianza menor y mayor al $95 \%$ fueron 23.29 y $35.27 \mu \mathrm{g} / \mathrm{L}$, respectivamente (Fig. 2, Cuadro IV).
CUADRO IV. CONCENTRACIÓN LETAL (CL) DE TALSTAR $®$ (I.C. $95 \%, p<0.01, R^{2}=82.07, x^{2}=$ 110.86) Y BIOTHRINE® (I.C. $95 \%, \mathrm{p}<0.01$, $\left.\mathrm{R}^{2}=81.82, x^{2}=127.18\right)$ EN Oreochromis niloticus

\begin{tabular}{ccc}
\hline $\begin{array}{c}\text { CL } \\
\%\end{array}$ & $\begin{array}{c}\text { Talstar }(\mu) \\
(\mu \mathrm{g} / \mathrm{L})\end{array}$ & $\begin{array}{c}\text { Biothrine }\left({ }^{\circledR}\right. \\
(\mu \mathrm{g} / \mathrm{L})\end{array}$ \\
\hline 15 &.-- & 3.95 \\
20 & 4.02 & 8.53 \\
25 & 18.14 & 12.47 \\
30 & 30.81 & 16.01 \\
35 & 42.56 & 19.28 \\
40 & 53.71 & 22.39 \\
45 & 64.50 & 25.40 \\
50 & 75.11 & 28.36 \\
55 & 85.73 & 31.32 \\
60 & 96.51 & 34.32 \\
65 & 107.66 & 37.43 \\
70 & 119.41 & 40.71 \\
75 & 132.09 & 44.24 \\
80 & 146.21 & 48.18 \\
85 & 162.66 & 52.77 \\
90 & 183.37 & 58.54 \\
95 & 214.06 & 67.10 \\
99 & 271.62 & 83.15 \\
\hline
\end{tabular}

I.C. = Intervalo de confianza, $\mathrm{R}^{2}=$ coeficiente de determinación, $x^{2}=$ chi cuadrada

CUADRO V. MORTALIDAD DE Oreochromis niloticus POR EFECTO DE BIOTHRINE® A DIFERENTES HORAS DE LECTURA

\begin{tabular}{ccccccccc}
\hline \multirow{2}{*}{$\begin{array}{c}\text { Tiempo } \\
\text { (h) }\end{array}$} & \multicolumn{7}{c}{ Concentración de Biothrine ${ }^{\circledR}(\mu \mathrm{g} / \mathrm{L})$} \\
\cline { 2 - 8 } & 0.56 & 1.4 & 2.8 & 7.0 & 14 & 28 & 56 & 112 \\
\hline 0.5 & 0 & 0 & 0 & 0 & 0 & 0 & 0 & 0 \\
1 & 0 & 0 & 0 & 0 & 0 & 0 & 0 & 0 \\
2 & 0 & 0 & 0 & 0 & 0 & 0 & 0 & 13 \\
3 & 0 & 0 & 0 & 0 & 0 & 0 & 0 & 13 \\
4 & 0 & 0 & 0 & 0 & 0 & 0 & 13 & 13 \\
5 & 0 & 0 & 0 & 0 & 0 & 0 & 13 & 25 \\
6 & 0 & 0 & 0 & 0 & 0 & 0 & 25 & 25 \\
7 & 0 & 0 & 0 & 0 & 0 & 13 & 25 & 38 \\
8 & 0 & 0 & 0 & 0 & 0 & 13 & 38 & 38 \\
9 & 0 & 0 & 0 & 0 & 0 & 25 & 38 & 38 \\
10 & 0 & 0 & 0 & 0 & 13 & 25 & 38 & 50 \\
11 & 0 & 0 & 0 & 0 & 13 & 25 & 38 & 50 \\
12 & 0 & 0 & 0 & 0 & 13 & 38 & 50 & 63 \\
18 & 0 & 0 & 0 & 0 & 25 & 38 & 50 & 63 \\
24 & 0 & 0 & 0 & 0 & 25 & 38 & 50 & 75 \\
36 & 0 & 0 & 0 & 0 & 38 & 50 & 63 & 75 \\
48 & 0 & 0 & 0 & 13 & 38 & 50 & 63 & 75 \\
60 & 0 & 0 & 0 & 13 & 38 & 50 & 63 & 75 \\
72 & 0 & 0 & 0 & 13 & 38 & 50 & 75 & 88 \\
84 & 0 & 0 & 13 & 25 & 50 & 63 & 75 & 88 \\
96 & 0 & 0 & 13 & 25 & 50 & 63 & 75 & 100 \\
\hline
\end{tabular}




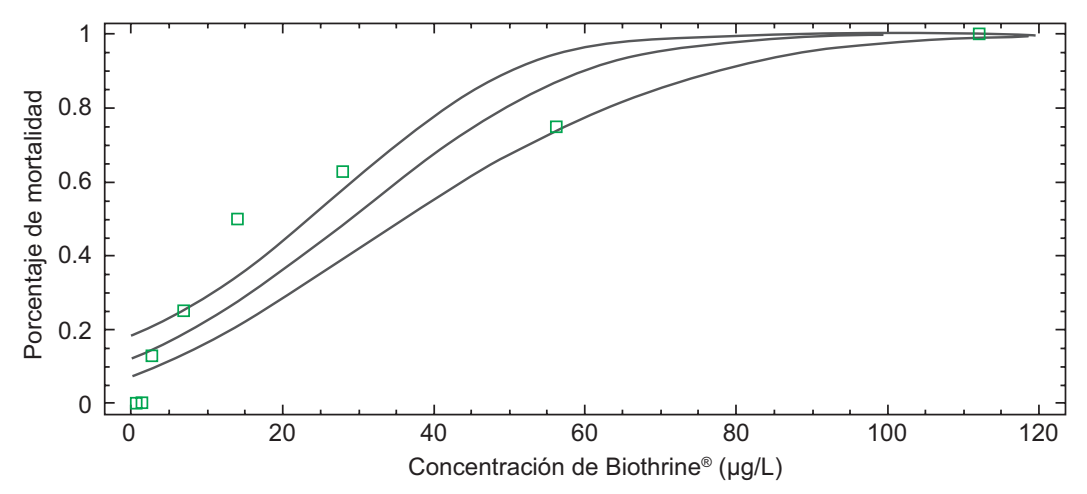

Fig. 2. Curva de la concentración letal 5096 h (I.C. $95 \%=23.29-35.27, \mathrm{p}<0.01$, $\mathrm{R}^{2}=81.82, x^{2}=127.18$ ) para Biothrine ${ }^{\circledR}$ en Oreochromis niloticus. I.C. $=$ Intervalo de confianza, $\mathrm{R}^{2}=$ coeficiente de determinación, $x^{2}=$ chi cuadrada

\section{DISCUSIÓN}

El cultivo de la tilapia está en desarrollo en el sureste de México, pero diversas acciones ponen en riesgo la inocuidad alimentaria de su producción. Una de ellas es el uso indiscriminado de insecticidas piretroides que el sector salud dispersa para hacer frente al mosquito vector del dengue (Sabra y Mehana 2015) y de otras enfermedades emergentes como chikungunya y zika (Gratz 2004, Musso y Nhan 2015).

La deltametrina y la bifentrina son los principios activos de un número cada vez mayor de insecticidas comerciales elegidos para estas fumigaciones con efectos tóxicos no evaluados lo suficiente en especies de importancia para la acuacultura, tanto en el sureste como en otras regiones de México (CENAPRECE 2016). Por ello, la determinación de sus grados de toxicidad se hace necesaria, en especial al incluir los datos de sus formulaciones comerciales (Yildirim et al. 2006, Monte et al. 2016).

Se obtuvo a las 96 horas en ambos insecticiadas, una CL50 de $75.11 \mu \mathrm{g} / \mathrm{L}$ para Talstar ${ }^{\circledR}$ y de $28.36 \mu \mathrm{g} / \mathrm{L}$ para Biothrine ${ }^{\circledR}$, estas formulaciones comerciales, contienen 91 y $97.5 \%$ de ingredientes surfactantes y solventes, respectivamente.

Velíšek et al. (2007), documentaron una CL50 de $0.02 \mathrm{mg} / \mathrm{L}$ a las 96 horas de contacto con el pesticida Decis EW $50 \AA$ (a base de deltametrina) en la trucha arcoíris (Oncorhynchus mykiss). Velíšek et al. (2009a) registraron una CL50 de $57.5 \mu \mathrm{g} / \mathrm{L}$ a las 96 horas en juveniles de carpa Cyprinus capio expuestas a Talstar ${ }^{\circledR}$. Ponepal et al. (2010), encontraron un gradiente de toxicidad para este insecticida comercial en percas (Perca fluviatilis) evaluadas a 96, 168 y 336 horas. Datos más recientes han registrado la CL50 de $8.7 \mu \mathrm{g} / \mathrm{L}$ a las 96 horas en Oreochromis mossambicus, expuestas a Trebon ${ }^{\circledR}$, el cual es un insecticida comercial cuyo principio activo es el piretroide Etofenprox, (Chandrasekara y Premarathna 2016).

A pesar de la falta de un patrón de referencia que permita realizar comparaciones directas de las CL50 obtenidas para Talstar ${ }^{\circledR}$ y Biothrine ${ }^{\circledR}$ con insecticidas comerciales a base de piretroides, incluyendo bifentrina y deltametrina, se puede deducir el riesgo tóxico que presentan esas formulaciones para los organismos acuáticos en la península de Yucatán. Esto, porque para el criterio de Sabra y Mehana (2015), los datos de CL50 obtenidos en este trabajo se sitúan dentro de una escala de peligro, específicamente en un rango extremadamente tóxico (valores entre 10 y $100 \mu \mathrm{g} / \mathrm{L}$ ).

Los piretroides producen baja toxicidad en mamíferos y aves, pero en los peces se presenta una mayor susceptibilidad, debido a un desequilibrio entre una alta tasa de absorción branquial y una baja detoxificación hidrolítica (Yang et al. 2014). En especial los peces tienen ciertas deficiencias enzimáticas para hidrolizar los piretroides, además su sistema nervioso es muy sensible y tienen una baja capacidad de metabolizar y eliminar insecticidas (Bradbury et al. 1986, Viran et al. 2003, Aydin et al. 2005).

Los efectos deletéreos en los piretroides se han podido diferenciar en dos grandes subdivisiones para los mecanismos de neurotoxicidad que pueden generar en mamíferos. En el caso de los piretroides tipo I, éstos producen un síndrome de intoxicación T, que se caracteriza por temblores, hiperexcitabilidad y ataxia locomotriz. En contraste, los piretroides tipo II, provocan el síndrome $\mathrm{C}$, donde se presenta coreo atetosis y salivación (Breckenridge et al. 2009). 
En este contexto, la bifentrina es un piretroide tipo I que altera los canales de sodio en las terminales de las células nerviosas para despolarizar el funcionamiento pre sináptico (de Moraes et al. 2013). Por otro lado, el mecanismo de toxicidad de la deltametrina piretroide tipo II, ocurre mediante el bloqueo de los canales de sodio de los filamentos nerviosos, lo que alarga la fase de despolarización y resulta en la afectación de los receptores del ácido $\gamma$-aminobutírico (GABA) en dicho tejido (El-Sayed y Saad 2007, Anadón et al. 2009).

Entre los daños evidenciados en peces para los piretroides tipo I, como la bifentrina en concentraciones del rango de $\mu \mathrm{g} / \mathrm{L}$, se encuentran alteraciones en la función motriz, cambios adversos en el desarrollo y muerte (Jin et al. 2010). Entre los efectos de los piretroides tipo II, como la deltametrina, se ha argumentado que también afectan la función motriz en concentraciones tan bajas del nivel de ng/L, además de producir efectos neurotóxicos, en el caso del pez cebra, puede reducir la fecundidad y la eclosión (De Micco et al. 2010, Sharma y Ansari 2010).

Se sabe que los piretroides tipo II son más tóxicos que los del tipo I, debido a que los piretroides del tipo II mantienen la inactivación de los canales de sodio durante un tiempo más largo que los compuestos tipo I, lo que imposibilita la generación del potencial de acción (Klaassen y Watkins III 2003, El-Sayed y Saad 2007).

En coincidencia con esta información, podemos destacar que para el presente estudio, el compuesto de Biothrine $\mathbb{R}$ (a base de deltametrina, piretroide tipo II) resultó más tóxico para la tilapia que Talstar ${ }^{\circledR}$ (a base de bifentrina, piretroide tipo I) en las condiciones experimentales establecidas.

En nuestro estudio, las principales afectaciones visibles en las tilapias expuestas a ambas mezclas de piretroides fueron: incremento de los movimientos operculares, pigmentación en la piel, pérdida del equilibrio y movimiento errático, siendo más conspicuas a una mayor concentración del insecticida administrado. Estas observaciones coinciden con lo encontrado por otros autores para exposiciones agudas y subagudas de piretroides en peces (El-Sayed y Saad 2007, Velíšek et al. 2009b). Es de resaltar que estas manifestaciones suelen ser irreversibles si el nivel de toxicidad supera el umbral máximo de exposición y acumulación (Herwig 1979).

Aunque los valores de la CL50 para Talstar ${ }^{\circledR}$ y Biothrine $\AA$ obtenidos en esta investigación son menores a los reportados por otros autores en cuerpos de agua para bifentrina y deltametrina, la exposición de piretroides en peces de ambientes naturales puede producirse por absorción branquial dada la alta lipofilicidad de los insecticidas o a través de la cadena alimenticia (Alonso et al. 2012).

En un estudio realizado en Nigeria, Akan et al. (2015) encontraron rangos de concentraciones entre 0.29 y $1.36 \mathrm{mg} / \mathrm{L}$ para bifentrina y entre 0.28 y $1.14 \mathrm{mg} / \mathrm{L}$ para deltametrina en agua de río. Ambas concentraciones estuvieron por debajo de los límites permisibles en aguas para la Unión Europea (100 y $50 \mathrm{mg} / \mathrm{L}$ para bifentrina y deltametrina, respectivamente).

En la península de Yucatán predominan datos sobre detección de plaguicidas organoclorados y organofosforados en agua y suelo, mientras que son escasos para los piretroides (Ponce-Caballero et al. 2012). A pesar de que se ha argumentado la posibilidad de contaminación de acuíferos por piretroides, las formulaciones comerciales comunes en programas de control de vectores incluyen surfactantes y agentes emulsificantes que podrían favorecer el transporte de los plaguicidas hasta los cuerpos subterráneos de agua y potencializar su persistencia en la cadena trófica (Pawlisz et al. 1998, Schleier III y Peterson 2011, Khalili et al. 2012, Akan et al. 2015).

Para el caso particular de los piretroides, su persistencia es muy corta y su solubilidad en agua es muy baja $(1-10 \mu \mathrm{g} / \mathrm{L})$, por lo que se podría sugerir un bajo potencial de contaminación acuática (Coats et al. 1989). No obstante, la forma más utilizada para fumigación y que se eligió en este estudio, es en polvo para hidratar lo que aumenta su disponibilidad en la cadena trófica y podría tener un potencial tóxico hasta nueve veces más que en su grado técnico (Neelima et al. 2016). Lo anterior aunado a un uso indiscriminado, aumenta el riesgo de exposición significativa en organismos de vida silvestre, domésticos o de importancia económica (De Micco et al. 2010, Goulding et al. 2013).

Así, se afirma que la toxicidad de los piretroides se incrementa con temperaturas bajas de agua (Kumaraguru y Beamish 1981, Oros y Werner 2005). Este efecto se atribuye a un mecanismo bioquímico de los peces basado en un aumento en el flujo de la corriente de sodio en las membranas sinápticas a bajas temperaturas (Narahashi et al. 1998).

En el contexto local, esta tendencia dependiente de la temperatura podría tener implicaciones para la acuacultura, ya que en la península de Yucatán prevalecen temperaturas calurosas, en promedio de 26 $\pm 1{ }^{\circ} \mathrm{C}$ (Vidal-Zepeda 2005). Esto podría incidir en que el potencial de toxicidad por los insecticidas sería de menor impacto para esa actividad, dado el riesgo disminuido por los altos valores de la temperatura ambiental prevaleciente en la región. 
Acorde a este planteamiento, y ya que la península de Yucatán se encuentra sobre una gran masa calcárea caracterizada por poseer niveles elevados de $\mathrm{CaCO}_{3}$ (Pérez y Pacheco 2004), también la dureza podría significar un factor amortiguador de la toxicidad por insecticidas piretroides. A este respecto, se conoce que este parámetro puede influir en la toxicidad de los piretroides (Mauck et al. 1976). Esto, porque existen indicios de que algunas enzimas involucradas en el transporte activo en las branquias pudieran inhibirse por los piretroides bajo la influencia de cambios en la dureza del agua y ocasionar un desbalance osmótico (Coats et al. 1989, Dyer et al. 1989). En este sentido, se requieren más estudios para corroborar la influencia de la temperatura y la dureza del agua en el grado de toxicidad por piretroides en peces.

Por último, el uso de piretroides en programas de control del dengue se prioriza por encima de otros insecticidas debido a su mayor fotoestabilidad, a su efectividad contra del mosquito vector y a su menor toxicidad para organismos como aves y mamíferos, pero se ha demostrado efectos nocivos mayores para peces (El-Sayed y Saad 2007). Una alternativa viable se dirige a evaluar la utilidad de extractos vegetales para el control del mosquito, ya que podrían ser menos tóxicos para los peces por considerarse biodegradables (Ghosh et al. 2012, Queen et al. 2014).

\section{CONCLUSIONES}

Este es el primer trabajo que evalúa los efectos tóxicos agudos individuales de insecticidas comerciales en especies piscícolas de importancia comercial en la península de Yucatán. Las CL50 de Talstar ${ }^{\circledR}$ (bifentrina) y de Biothrine ${ }^{\circledR}$ (deltametrina) a las 96 horas en $O$. niloticus fueron de $75.11 \mu \mathrm{g} / \mathrm{L}$ y $28.36 \mu \mathrm{g} / \mathrm{L}$, respectivamente. Los signos de toxicidad en los peces bajo influencia de ambos insecticidas fueron movimientos erráticos, desbalance e hiperventilación, siendo más evidentes y en menor tiempo, en concentraciones más altas.

Los valores de CL50 obtenidos se consideran extremadamente tóxicos, por lo que se debe alertar dado el potencial efecto contaminante que se podría producir en ambientes naturales como los prevalecientes en la península, así como en condiciones de acuacultura.

Es posible que estos insecticidas no tengan un alto impacto negativo en condiciones de campo dada su mínima adsorción en agua, así como por el amortiguamiento de la temperatura y de la dureza del agua. Aunque la alta lipofilicidad de estos piretroides promueve el potencial de absorción en el tejido adiposo. Además, es importante reconocer que los concentrados emulsionables que se utilizan en estos plaguicidas en el sector salud, favorecen el tiempo de permanencia en la cadena trófica. Los datos obtenidos en este trabajo mediante condiciones controladas, podrían ser de utilidad para estudios futuros que evalúen los efectos de los plaguicidas piretroides, ya sea de formulaciones comerciales o de sus principios activos, en ecosistemas similares a los de la península de Yucatán.

Los datos obtenidos reafirman la necesidad de continuar con la evaluación de los mecanismos de toxicidad de sustancias utilizadas en el sector salud sobre especies piscícolas ya que se tiene la perspectiva de continuar con su uso, aunque el desarrollo del sector en la región prevalezca.

\section{AGRADECIMIENTOS}

Los resultados de esta investigación son parte de los proyectos: "Caracterización toxicológica en peces de importancia comercial como modelo para evaluar los efectos de contaminantes agroquímicos" (clave TIZ-MCAS-2013-249) de la Dirección General de Educación Superior Tecnológica (DGEST) y "Efectos tóxicos en un sistema de cultivo sustentable tilapia-lombriz de tierra (Oreochromis niloticusEisenia foetida) expuesto a plaguicidas piretroides" (clave 5877.16-P) del Tecnológico Nacional de México (TecNM).

\section{REFERENCIAS}

Akan J.C., Battah N., Waziri M. y Mahmud M.M. (2015). Organochlorine, organophosphorus and pyrethroid pesticides residues in water and sediment samples from River Benue in Vinikilang, Yola, Adamawa state, Nigeria using gas Chromatography-Mass Spectrometry equipped with Electron Capture Detector. Am. J. Environ. Protect. 3 (5), 164-173.

DOI: 10.12691/env-3-5-2

Alonso M.B., Feo M.L., Corcellas C., Vidal L.G., Bertozzi C.P., Marigo J., Secchi E.R., Bassoi M., Azevedo A.F., Dorneles P.R., Torres J.P.M., Lailson-Brito J., Malm O., Eljarrat E. y Barcelo D. (2012). Pyrethroids: A new threat to marine mammals? Environ. Int. 47, 99-106. DOI: $10.1016 /$ j.envint.2012.06.010

Altagracia M.M., Kravzov J.J., Moreno B.C., López N.F. y Martínez N.J.M. (2012). Las enfermedades "olvidadas" de América Latina y el Caribe: un problema 
de salud pública global. Rev. Mex. Cienc. Farm. 43 (1), 33-41.

Anadón A., Martínez L.M.R. y Martínez M.A. (2009). Use and abuse of pyrethrins and synthetic pyrethroids in veterinary medicine. Vet. J. 182 (1), 7-20.

DOI: $10.1016 /$ j.tvjl.2008.04.008

Antwi F.B. y Reddy G.V.P. (2015). Toxicological effects of pyrethroids on non-target aquatic insects. Environ. Toxicol. Pharmacol. 40 (3), 915-923.

DOI: $10.1016 /$ j.etap.2015.09.023

Aydin R., Köprücü K., Dörücü M., Köprücü S.S. y Pala M. (2005). Acute toxicity of synthetic pyrethroid cypermethrin on the common carp (Cyprinus carpio L.) embryos larvae. Aquat. Int. 13 (5), 451-458.

DOI: $10.1007 / \mathrm{s} 10499-005-0615-5$

Bálint T., Szegletes T., Szegletes Z., Halasy K. y Nemcsók J. (1995). Biochemical and subcellular changes in carp exposed to organophosphorus methidathion and the pyrethroid deltamethrin. Aquat. Toxicol. 33 (3-4), 279-295. DOI: 10.1016/0166-445X(95)00029-4

Barba E.J.R. (2014). Dengue. Problema que preocupa y ocupa a la salud pública. Rev. Latinoam. Patol. Clin. Med. Lab. 61 (2), 84-101.

Başer S., Erkoç F., Selvi M. y Koçak O. (2003). Investigation of acute toxicity of permethrin on guppies Poecilia reticulate. Chemosphere 51 (6), 469-474. DOI: 10.1016/S0045-6535(03)00033-X

Boateng J.O., Nunoo F.K.E., Dankwa H.R. y Ocran M.H. (2006). Acute toxic effects of deltamethrin on tilapia, Oreochromis niloticus (Linnaeus, 1758). West Afr. J. Appl. Ecol. 9 (1), 1-5. DOI: 10.4314/wajae.v9i1.45661

Bradbury S.P., Coats J.R. y McKim J.M. (1986). Toxicokinetics of fenvalerate in rainbow trout (Salmo gairdneri). Environ. Toxicol. Chem. 5 (6), 567-576.

Brander S.M., Gabler M.K., Fowler N.L., Connon R.E. y Schlenk D. (2016). Pyrethroid pesticides as endocrine disruptors: molecular mechanisms in vertebrates with a focus on fishes. Environ. Sc. Technol. 50 (17), 89778992. DOI: 10.1021/acs.est.6b02253

Breckenridge C.B., Holden L., Sturgess N., Weiner M., Sheets L., Sargent D., Soderlund D.M., Choi J.S., Symington S., Marshall C.J., Burr S. y Ray D. (2009). Evidence for a separate mechanism of toxicity for the Type I and the Type II pyrethroid insecticides. NeuroToxicology 30, S17-S31.

DOI: 10.1016/j.neuro.2009.09.002

Buckley D.K., MacDonald D.M.J., Villasuso M., Graniel E., Vázquez J. y Jiménez M. (1994). Geophysical logging of a karstic limestone aquifer for hydrogeological purposes at Merida, Yucatan, Mexico. Reporte Técnico 194/4C, British Geological Survey, 40 pp.

CENAPRECE (2016). Lista actualizada de productos recomendados por el Centro Nacional de Programas
Preventivos y Control de Enfermedades (CENAPRECE) para el combate de insectos vectores de enfermedades a partir de 2016 [en línea]. http://www. cenaprece.salud.gob.mx/programas/interior/vectores/ descargas/pdf/ListaActualizadaProductoRecomendadosCENAPRECE2016_1.pdf 18/10/2016

CONAPESCA (2013). Anuario estadístico de acuacultura y pesca. Comisión Nacional de Acuacultura y Pesca [en línea]. http://www.gob.mx/conapesca/documentos/anuario-estadistico-de-acuacultura-y-pesca 27/03/2016

Coats J.R., Symonik D.M., Bradbury S.P., Dyer S.D., Timson L.K. y Atchison G.J. (1989). Toxicology of synthetic pyrethroids in aquatic organisms: an overview. Environ. Toxicol. Chem. 8 (8), 671-679.

Chandrasekara W.U. y Premarathna K.S.D. (2016). Toxic effects of Trebon $\AA$, a synthetic pyrethroid based insecticide formulation, on Oreochromis mossambicus (Family: Cichlidae). Sri Lanka Journal of Aquatic Science 21 (1), 35-49. DOI: 10.4038/sljas.v21i1.7484

De Micco A., Cooper K.R., Richardson J.R. y White L.A. (2010). Developmental neurotoxicity of pyrethroid insecticides in zebrafish embryos. Toxicol. Sci. 113 (1), 177-186. DOI: 10.1093/toxsci/kfp258

de Moraes F.D., Venturini F.P., Cortella L.R.X., Rossi P.A. y Moraes G. (2013). Acute toxicity of pyrethroidsbased insecticides in the Neotropical freshwater fish Brycon amazonicus. Ecotoxicol. Environ. Contam. 8 (2), 59-64. DOI: 10.5132/eec.2013.02.009

Dobsikova R., Velisek J., Wlasow T., Gomulka P., Svobodova Z. y Novotny L. (2006). Effects of cypermethrin on some haematological, biochemical and histopathological parameters of common carp (Cyprinus carpio L.). Neuroendocrinol. Lett. 27 (2), 91-95.

Dyer S.D., Coats J.R., Bradbury S.P., Atchison G.J. y Clark J.M. (1989). Effects of hardness and salinity on the acute toxicity and uptake of fenvalerate by bluegill (Lepomis macrochirus). Bull. Environ. Contam. Toxicol. 42 (3), 359-366.

DOI: $10.1007 / \mathrm{BF} 01699961$

El-Sayed Y.S. y Saad T.T. (2007). Subacute intoxication of a deltamethrin-based preparation (Butox ${ }^{\circledR} 5 \%$ EC) in monosex Nile tilapia, Oreochromis niloticus L. Basic Clin. Pharmacol. Toxicol. 102 (3), 293-299. DOI: 10.1111/j.1742-7843.2007.00157.x

Finney D.J. (1971). Probit analysis. 3a ed. Cambridge University Press. Nueva York, EUA, 333 pp.

Ghosh A., Chowdhury N. y Chandra G. (2012). Plants extracts as potential mosquito larvicides. Indian J. Med. Res. 135, 581-598.

González O.G. (2013). Resistencia a insecticidas en el mosquito vector del dengue Aedes aegypti (L) en dos épocas de transmisión de la enfermedad en Mérida, Yucatán. Tesis de Doctorado. Universidad Autónoma de 
Nuevo León. Monterrey, Nuevo León, México, 145 pp.

Goulding A.T., Shelley L.K., Ross P.S. y Kennedy C.J. (2013). Reduction in swimming performance in juvenile rainbow trout (Oncorhynchus mykiss) following sublethal exposure to pyrethroid insecticides. Comp. Biochem. Physiol. C 157 (3), 280-286.

DOI: $10.1016 /$ j.cbpc.2013.01.001

Gratz N.G. (2004). Critical review of the vector status of Aedes albopictus. Med. Vet. Entomol. 18 (3), 215-227. DOI: 10.1111/j.0269-283X.2004.00513.x

Gradish A.E., Scott-Dupree C.D., Frewin A.J. y Cutler G.C. (2012). Lethal and sublethal effects of some insecticides recommended for wild blueberry on the pollinator Bombus impatiens. Can. Entomol. 144 (3), 478-486.

DOI: $10.4039 /$ tce. 2012.40

Hedayati A., Miandareh H.K., Shaluei F. y Jahanbakhshi A. (2012). Toxicity responses of silver carp (Hypophthalmichthys molitrix) and roach (Rutilus rutilus) during lethal exposure to deltamethrin. J. Comp. Clin. Path. Res. 1/2, 47-51.

Herwig N. (1979). Handbook of drugs and chemicals used in the treatment of fish diseases. Charles $\mathrm{C}$. Thomas Publisher, Springfield, Illinois, EUA, 272 pp.

Jahan N. y Sadiq A. (2012). Evaluation of resistance against bifenthrin in dengue vector from Lahore, Pakistan. Biol. Pak. 58 (1-2), 13-19.

Jin M., Zhang Y., Ye J., Huang C., Zhao M. y Liu W. (2010). Dual enantioselective effect of the insecticide bifenthrin on locomotor behavior and development in embryonic-larval zebrafish. Environ. Toxicol. Chem. 29 (7), 1561-1567. DOI: 10.1002/etc. 190

Khalili M., Khaleghi S.R. y Hedayati A. (2012). Acute toxicity test of two pesticides diazinon and deltamethrin, on swordtail fish (Xiphophorus helleri). Glob. Vet. 8 (5), 541-545.

Karasu B.A.Ç.K., Selvi M., Sarikaya R., Erkoç F. y Koçak O. (2009). Acute toxicity of deltamethrin on Nile tilapia (Oreochromis niloticus L.1758) Larvae and Fry. G.U. J. Sc. $22(1), 1-4$.

Kidd H. y James D.R. (1991). The agrochemicals handbook. 3a ed. Lewis Publishers/Royal Society of Chemistry Information Services. Cambridge, Inglaterra, 1500 pp.

Klaassen C.D. y Watkins III J.B. (2003). Casarett y Doull fundamentos de toxicología. McGraw Hill/Interamericana, Madrid, España, 536 pp.

Kumaraguru A.K. y Beamish F.W.H. (1981). Lethal toxicity of permethrin (NRDC-143) to rainbow trout (Salmo gairdneri) in relation to body weight and water temperature. Water Res. 15 (4), 503-505.

DOI: 10.1016/0043-1354(81)90061-0

Liu T.L., Wang Y.S. y Yen. J.H. (2005). Separation of bifenthrin enantiomers by chiral HPLC and determi- nation of their toxicity to aquatic organisms. J. Food Drug Anal. 13 (4), 357-360.

Mauck W.L., Olson L.E. y Marking L.L. (1976). Toxicity of natural pyrethrins and five pyrethroids to fish. Arch. Environ. Con. Tox. 4 (1), 18-29.

DOI: $10.1007 / \mathrm{BF} 02221012$

Montanha F.P., Galeb L.A., Mikos J.D., Ganeco L.N., Pereira T.P., Tanaka A., Kirschnik P.G. y Pimpão C.T. (2012). Pyrethroid toxicity in silver cat_ish, Rhamdia quelen. Pesq. Vet. Bras. 32 (12), 1297-1303.

DOI: $10.1590 / \mathrm{S} 0100-736 \mathrm{X} 2012001200014$

Monte T.C.C., García J., Gentile R., de Vasconcellos M.C, Souza J., Braga B.V. y Maldonado A. (2016). In vivo and in vitro effects of the herbicide Roundup ${ }^{\circledR}$ on developmental stages of the trematode Echinostoma paraensei. Exp. Parasitol. 169, 43-50.

DOI: 10.1016/j.exppara.2016.06.012

Musso D. y Nhan T.X. (2015). Emergence of zika virus. Clin. Microbiol. 4 (5), 1-4. DOI: $10.4172 / 2327-5073.1000222$

Narahashi T., Ginsburg K.S., Nagata K., Song J.H. y Tatebayashi H. (1998). Ion channels as targets for insecticides. Neurotoxicology. 19 (4-5), 581-90.

Neelima P., Rao K.G., Rao G.S., Rao N.G. y Rao J.C.S. (2016). Biomarkers of cypermethrin (synthetic pyrethroid) toxicity - biochemical alterations in Cyprinus carpio, a freshwater edible fish. International J. Biol. Med. Res. 7 (2), 5574-5581.

Olvera N.M. (2010). La acuacultura y su posible impacto en la biodiversidad. En: Biodiversidad y desarrollo en Yucatán (R. Durán y M. Méndez, Eds.) Centro de Investigación Científica de Yucatán, Programa de Pequeñas Donaciones del Fondo para el Medio Ambiente Mundial, Comisión Nacional para el Conocimiento y Uso de la Biodiversidad, Secretaría de Desarrollo Urbano y Medio Ambiente Yucatán. Mérida, Yucatán, México, pp. 116-118.

Oros D.R. y Werner I. (2005). Pyrethroid insecticides: an analysis of use patterns, distributions, potential toxicity and fate in the Sacramento-San Joaquin Delta and Central Valley. White Paper for the Interagency Ecological Program. SFEI Contribution 415. San Francisco Estuary Institute, Oakland, EUA, 113 pp.

Pacheco-Ávila J., Cabrera-Sansores A. y Pérez-Ceballos R. (2004). Diagnóstico de la calidad del agua subterránea en los sistemas municipales de abastecimiento en el Estado de Yucatán, México. Ingeniería 8, 165-179.

Pawlisz A.V., Busnarda J., McLauchlin A., Caux P.Y. y Kent R.A. (1998). Canadian water quality guidelines for deltamethrin. Environ. Toxicol. Water Qual. 13 (3), 175-210.

DOI: 10.1002/(SICI)1098-2256(1998)13:3<175::AIDTOX1>3.0.CO;2-4 
Pérez C.R. y Pacheco A.J. (2004). Vulnerabilidad del agua subterránea a la contaminación de nitratos en el estado de Yucatán. Ingeniería 8, 33-42.

Ponce-Caballero C., Castellanos-Mercado R.M. y CoronaCruz A. (2012). Plaguicidas en el sureste de México: problemática ambiental, de salud, tecnología de tratamiento y perspectivas. En: Contribución de la biotecnología al desarrollo de la península de Yucatán. (E. Dumonteil, Ed.). Consejo de Ciencia, Innovación y Tecnología del Estado de Yucatán. Mérida, Yucatán, México, pp. 231-248.

Ponepal M.C., Păunescu A., Drăghici O. y Marinescu A.G. (2010). Research on the changes of some physiological parameters in several fish species under the action of the talstar insecticide. An. UO. Fasc. Biol. 17, 175-179.

Prusty A.K., Meena D.K., Mohapatra S., Panikkar P., Das P., Gupta S.K. y Behera B.K. (2015). Synthetic pyrethroids (Type II) and freshwater fish culture: Perils and mitigations. Int Aquat Res. 7 (3), 163-191.

DOI: 10.1007/s40071-015-0106-X

Queen M.M., Martin P., Elumalai K., Kandeepan C., Kalaimathi R.V. y Sivamani P. (2014). Effect of selected plant fumigants against dengue fever vector Aedes aegypti (Linn). Int. J. Curr. Res. Aca. Rev. 2 (8), 294-303.

Reish L.D. y Oshida J.P. (1987). Manual of methods in aquatic environment research. Part 10- Short term static bioassays. Food and Agriculture Organization of the United Nations. Roma, Italia, 64 pp.

Sabra F.S. y Mehana E.S.E.D. (2015). Pesticides toxicity in fish with particular reference to insecticides. Asian J. Agr. Food Sci. 3 (1), 40-60.

Salomón G.J., Lugo M.G.V., Tinal G.V.R., de La Cruz V.J., Beaty B.J., Eisen L., Lozano F.S., Moore C.G. y García R.J.E. (2012). Aedes albopictus mosquitoes, Yucatan peninsula, Mexico. Emerg. Infect. Dis. 18 (3), 525-526.

Sarikaya R. (2009). Investigation of acute toxicity of alpha-cypermethrin on adult Nile tilapia (Oreochromis niloticus L.). Turk. J. Fish. Aquat. Sc. 9 (1), 85-89.

Schleier III J.J. y Peterson R.K.D. (2011). Pyrethrins and pyrethroi insecticides. En: Green trends in insect control (O. López y J. G. Fernández-Bolaños, Eds.). Royal Society of Chemistry. Londres, Inglaterra, pp. 94-131.

Sharma D. y Ansari B. (2010). Effect of the synthetic pyrethroid deltamethrin and the neem-based pesticide achook on the reproductive ability of zebrafish, Danio rerio (Cyprinidae). Arch. Pol. Fish. 18 (3), 157-161. DOI: $10.2478 / \mathrm{v} 10086-010-0017-9$

SSA (2010). Norma Oficial Mexicana NOM-032SSA2-2010, Para la vigilancia epidemiológica, prevención y control de las enfermedades transmitidas por vector. Secretaría de Salud. Diario Oficial de la Federación. 01 de junio de 2011.

Velíšek J., Jurčíková J., Dobšíková R., Svobodová Z., Piačková V., Máchová J. y Novotný L. (2007). Effects of deltamethrin on rainbow trout (Oncorhynchus mykiss). Environ. Toxicol. Pharmacol. 23 (3), 297-301. DOI: 10.1016/j.etap.2006.11.006

Velíšek J., Svobodová Z. y Máchová J. (2009a). Effects of bifenthrin on some haematological, biological and histopathological parameters of common carp (Cyprinus carpio L.). Fish Physiol. Biochem. 35 (4), 583-590. DOI: 10.1007/s10695-008-9258-6

Velíšek J., Svobodová Z. y Piačková V. (2009b). Effects of acute exposure to bifenthrin on some haematological, biochemical and histopathological parameters of rainbow trout (Oncorhynchus mykiss). Vet. Med. 54 (3), 131-137.

Vidal-Zepeda R. (2005). Las regiones climáticas de México 1.2.2. Temas selectos de geografía de México. Instituto de Geografía, Universidad Nacional Autónoma México, Ciudad de México, México, 217 pp.

Viran R., Erkoç F. U., Polat H. y Koçak O. (2003). Investigation of acute toxicity of deltamethrin on guppies (Poecilia reticulata). Ecotoxicol. Environ. Saf. 55 (1), 82-85. DOI: 10.1016/S0147-6513(02)00096-9

Yang Y., Ma H., Zhou J., Liu J. y Liu W. (2014). Joint toxicity of permethrin and cypermethrin at sublethal concentrations to the embryo-larval zebrafish. Chemosphere 96, 146-154.

DOI: 10.1016/j.chemosphere.2013.10.014

Weston D.P., Aspell A.M., Hecht S.A., Scholz N.L. y Lydy M.J. (2011). Pyrethroid insecticides in urban salmon streams of the Pacific Northwest. Environ. Pollut. 159 (10), 3051-3056. DOI: 10.1016/j.envpol.2011.04.008 Yildirim M.Z., Karasu Benlı A.C. Selv1 M., Özkul A., Erkoç F. y Koçak O. (2006). Acute toxicity, behavioral changes, and histopathological effects of deltamethrin on tissues (gills, liver, brain, spleen, kidney, muscle, skin) of Nile tilapia (Oreochromis niloticus L.) fingerlings. Environ. Toxicol. 21 (6), 614-620.

DOI: $10.1002 /$ tox.20225

Zapata-Pérez O. y Pedrero Ríos J.M. (2008). Ensayo de toxicidad aguda con larvas y juveniles de los peces tilapia, carpa y cíclidos. En: Ensayos toxicológicos para la evaluación de sustancias químicas en agua y suelo. La experiencia en México. (A. Mendoza y P. Ramírez, Eds.). Instituto Nacional de Ecología y Cambio Climático, Ciudad de México, México, pp. 155-166. 